\title{
Finding hotspots by location-direction-enabled photographs focusing on intersection of lines of sight
}

\author{
Hideyuki Fujita $^{\text {a, }}$, Shota Sagara ${ }^{\text {b }}$, Tadashi Ohmori ${ }^{\text {a }}$, Takahiko Shintani ${ }^{\text {a }}$ \\ ${ }^{a}$ The University of Electro-Communications, fujita@is.uec.ac.jp, omori@is.uec.ac.jp, shintani@is.uec.ac.jp \\ ${ }^{b}$ NTT Resonant, Inc, sagara@nttr.co.jp \\ * Corresponding author
}

\begin{abstract}
We propose a method to extract a set of viewpoint locations and a set of locations of interest from a given set of location-direction-enabled photographs having embedded information regarding the location and direction of the shot. A viewpoint location is a location from which many photographs are taken. A location of interest is a location that that many photographs are taken of. When extracting locations of interest, a location-direction-enabled photograph does not embed the location of its subject; this is a technical difficulty. Therefore, we treat location-direction-enabled photographs as a point-with-directions and propose a novel clustering method for them. The evaluation results show that the method improves precision and recall for extracting locations of interest compared to an existing method. We also show that the proposed method can extract locations of interest that the existing method cannot extract.
\end{abstract}

Keywords: Location-direction-enabled photograph, Clustering, Hotspot, Viewpoint location, Location of interest

\section{Introduction}

We propose a method of extracting two types of hot spots named spot of viewpoint and interest spot for a set of location-direction-enabled photographs that contain information about the photographing direction (orientation of the camera at the time of photographing) in addition to the photographing location. The former is a place where many photographs are taken, and the latter is a place that is a subject in many photographs.

The problem is that, while a location-direction-enabled photograph has a location (shooting location) and a direction (shooting direction), it does not have the location information of its subject; therefore, it is not possible to extract a location of interest where the location information of the subject is accumulated. In this study, we treat the location-direction-enabled photograph as a directional data point with location and direction information and propose a clustering method for the directional data point, through which we extract spots of interest.

\section{Definition of the problem}

We define key concepts and objectives.

Directional point: An oriented point $p=(l o c, d i r)$ is the combination of 2-dimensional position coordinates loc and real value direction angle dir. The half line from loc to dir is called the line of sight.

Location-direction-enabled photograph: A photograph with information on the shooting position (position of the camera, denoted as $l o c$ ) and shooting direction (direction that the camera is aimed at, denoted as dir). Therefore, the directional point $p=(l o c, d i r)$.
Hotspot: A general term for spots of viewpoint (areas where shooting positions are concentrated) and spots of interest (areas of which many photographs are taken). Extracted as a point group in the 2-dimensional space cluster.

Using these definitions, the objective in this paper may be defined as follows:

- To extract sets of spots of viewpoint and interest spots from the given set of location-directionenabled photographs

\section{Overview of the method}

The spot of viewpoint is defined as a cluster of shooting positions for the given set $P$ of location-direction-enabled photographs. DBSCAN (Ester et al., 1996) is used for clustering.

The spot of interest is defined as the set $X$ consisting of the cluster of points of intersection of any two lines of sight of $P$ (Figure. 1). This is based on the assumption that the subject is in the vicinity of the point of intersection of 2 lines of sight aiming at it. However, if we consider the intersections of arbitrary pairs of lines, there may be many intersections that are distant from the viewpoint. This results in clusters that are unsuitable as interest spots being formed. We therefore suggest the use of dirDBSCAN for clustering instead of DBSCAN, it will help suppress such inadequate clusters. In this method, the neighbouring points in DBSCAN are assigned a weight of $[0,1]$ to suppress clusters. 


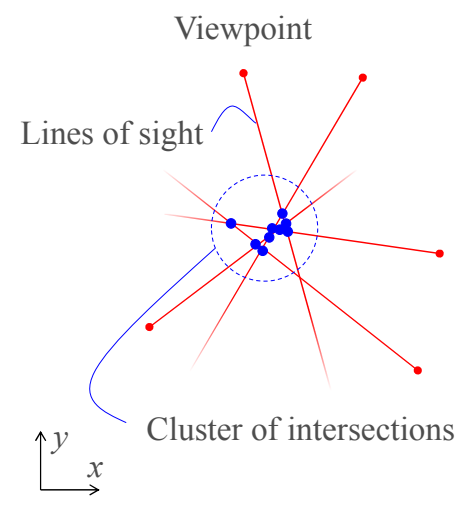

Figure 1. Clustering of intersections of lines-of-sight

\section{dirDBSCAN Method}

Using an arbitrary $x \in X$ as a seed, we extract as one cluster the largest set among those of transitively reachable subjects that satisfy the direct weighted density - reachable condition defined below.

\section{Definition Neighborhood}

The neighborhood $N_{E P S}\left(x_{p}\right)$ of $x_{p} \in X$ is defined as follows:

$$
N_{E P S}\left(x_{p}\right)=\left\{x_{q} \in X \mid x_{p} \neq x_{q}, \operatorname{Dist}\left(x_{p}, x_{q}\right) \leq E p s\right\}
$$

The function $\operatorname{Dist}\left(x_{p}, x_{q}\right)$ returns the distance between $x_{p}$ and $x_{q} . E_{p s}$ is the distance threshold.

\section{Definition directly weighted-density-reachable}

When $x_{p}, x_{q} \in X$, Eps, MinPts and $\sigma$ satisfy the following conditions, the distance from $x_{p}$ to $x_{q}$ is said to be directly weighted-density-reachable with respect to Eps, MinPts and $\sigma$.

$$
\begin{aligned}
& S_{E p s, \sigma}\left(x_{p}\right) \geq \text { MinPts } \\
& x_{q} \in N_{E p s}\left(x_{p}\right)
\end{aligned}
$$

MinPts is the threshold value for the number of intersection points. $S_{E p s, \sigma}\left(x_{p}\right)$ is the number of weighted intersection points in the neighborhood of $x_{p}$ and is derived as follows:

$$
\begin{aligned}
& S_{E p s, \sigma}\left(x_{p}\right)= \\
& S_{E p s}^{(\text {diversity) }}\left(x_{p}\right) \sum_{x \in N_{E p s}\left(x_{p}\right)} S^{(\text {angle })}(x) S_{E p s, \sigma}^{(\text {length })}(x)
\end{aligned}
$$

The purpose of the terms $S^{(\text {angle })}(x), S_{E p s, \sigma}^{(\text {length })}(x)$ and $S_{E p s}^{(\text {diversity })}(x)$ of this equation is stated below.

\section{Suppression of the intersection of a group of sight lines that are nearly parallel}

The score $S^{\text {(angle) }}(x)$ for the angle at intersection between two lines of sight is defined below for each intersection point $x$. The range of values is $[0,1]$.

$$
S^{(\text {angle })}(x)=\sin (\text { crossingAngle }(x))
$$

The function crossingAngle $(x)$ returns the angle at intersection between the two lines of sight that intersect at $x$.
Suppression of points of intersection that are distant from the viewpoint

The score $S_{E p s, \sigma}^{(\text {length }}(x)$ for the length of two lines of sight is defined below for each point of intersection $x$. The range of values is $[0,1]$.

$$
\begin{aligned}
& S_{E p s, \sigma}^{(\text {length })}(x)= \\
& \max \left\{0,1-\left(\frac{\max (\{\operatorname{dist}(p . \operatorname{loc}, x) \mid p \in \operatorname{org}(x)\})}{\sigma E p s}\right)^{2}\right\}
\end{aligned}
$$

$\sigma$ is a parameter that defines the threshold value for the length of the line of sight.

Suppression of clusters formed by concentration of intersection points where a majority of lines of sight comes from the same direction

The score $S_{E p s}^{\text {(diversity) }}(x)$ for the relationship between number of intersection points and number of lines of sight is defined for each intersection point $x$ as shown below. The range of values is $[0,1]$.

$$
S_{\text {Eps }}^{\text {(diversity) }}(x)=\frac{\left|N_{E p s}(x)\right|}{\text { Combination }\left(\left|\mathrm{U}_{x \prime \in N_{E p s}(x)} \operatorname{org}\left(x^{\prime}\right)\right|, 2\right)}
$$

The function $\operatorname{org}\left(x^{\prime}\right)$ returns a set of 2 lines of sight that intersect at $x^{\prime}$. Combination $(a, b)$ denotes the number of combinations for selecting a number of $b$ items from $a$ items.

\section{Evaluation of the effectiveness of each score}

The usefulness of each score from dirDBSCAN is evaluated using a simulation. In the object space, a circular area is defined as a spot of interest and a group of location-direction-enabled photographs is randomly generated such that the spot and the line of sight intersect. The possibility of extracting clusters by the proposed method using these spots as input is verified. The spatial constraints involved when generating a group of photographs are varied and combined with the application or nonapplication of each of the 3 types of scores in dirDBSCAN and the results are compared. To give a specific example, dirDBSCAN is achieved by generating $n$ sets of location-direction-enabled photographs for each combination of the pair $(\tau, \varphi)$ and the applied score $S_{a p p}$ based on conditions shown in Tables 1 and 2, and the number of clusters is determined. The result is the average value for $N$ trials of each combination. $S_{a p p}$ denotes the combination of scores applied in dirDBSCAN. As shown in equation (4), three types of scores $S^{(\text {angle })}(x), S_{E p s, \sigma}^{(\text {length })}(x)$ and $S_{E p s}^{(\text {diversity) }}(x)$ (abbreviated as div, ang, len) are used in dirDBSCAN. Experiments are conducted on all combinations (eight types shown in Table 1), and in each case a score may or may not be applied. When a score is not applied, the value of the corresponding score is set to 1 in equation (4). For example, in the case of $S_{a p p}=\{d i v, a n g\}$, div and ang are calculated and applied; hence, len $=1$.

The results are shown in Figure 2. Figures (a) - (g) correspond with the applied score set $S_{a p p}$. In the graphs, the $x$ axis is $\varphi$, the $y$ axis is the average number of 
clusters, and the broken lines correspond to $\tau$. The aim for the proposed method is to extract clusters while excluding those where the angular range is small or the distance between the shooting position and the viewpoint is large. The results show that $(\mathrm{h})$, where all scores are applied, is the best, while the generation of clusters is substantially suppressed when $\varphi=30^{\circ}$ or less, and with regard to the distance between the shooting position and the viewpoint, cluster generation with $\tau=8$ or more is substantially suppressed when $\sigma=10$.

\begin{tabular}{|c|c|}
\hline Parameter type & $\begin{array}{l}\text { Symbols and possible } \\
\text { values }\end{array}$ \\
\hline \multicolumn{2}{|l|}{ Constants } \\
\hline \begin{tabular}{l|l} 
Radius of spot of \\
interest
\end{tabular} & $r_{s}=1$ \\
\hline $\begin{array}{l}\text { Number of location- } \\
\text { direction-enabled } \\
\text { photographs }\end{array}$ & $n=8$ \\
\hline $\begin{array}{l}\text { dirDBSCAN } \\
\text { Parameter }\end{array}$ & $\begin{array}{l}(\text { Eps }, \text { MinPts }, \sigma) \\
=\left(r_{s}, 4,10\right)\end{array}$ \\
\hline $\begin{array}{l}\text { Number of trials in } \\
\text { each combination of } \\
\text { variables }\end{array}$ & $N=100$ \\
\hline \begin{tabular}{lr}
\multicolumn{2}{l}{ Distribution } \\
shooting position \\
viewpoint distance \\
(Ratio relative to \\
average)
\end{tabular} & $\rho=0.5$ \\
\hline
\end{tabular}

\begin{tabular}{l|l|l}
\hline \multicolumn{2}{l}{ Variables } \\
\hline $\begin{array}{l}\text { Shooting position / } \\
\text { viewpoint distance } \\
\text { (Ratio relative to the } \\
\text { radius of the spot of } \\
\text { interest) }\end{array}$ & $\tau=2,3,4, \ldots, 10$ \\
\hline $\begin{array}{l}\text { Upper limit of angular } \\
\text { range of shooting } \\
\text { direction }\end{array}$ & $\varphi=30,60,90, \ldots, 360^{\circ}$ \\
\hline $\begin{array}{l}\text { Combination of } \\
\text { applied scores }\end{array}$ & $\begin{array}{l}S_{\text {app }}=\{\},\{\text { ang }\},\{\text { div }\}, \\
\{\text { len }\},\{\text { div, ang }\},\{\text { div, len }\}, \\
\{\text { ang, len }\},\{\text { div, ang, len }\}\end{array}$ \\
\hline
\end{tabular}

Table 1. Parameters of simulation

\begin{tabular}{l|l}
\hline Spot of interest & $\begin{array}{l}\text { Circular region with center at the } \\
\text { origin and radius } r_{s}\end{array}$ \\
\hline $\begin{array}{l}\text { Location of } \\
\text { viewpoint } g\end{array}$ & $\begin{array}{l}\text { Generate within the spot of interest } \\
\text { using the coordinates of the } \\
\text { uniform random number }\end{array}$ \\
\hline $\begin{array}{l}\text { Shooting } \\
\text { direction } p . d i r\end{array}$ & $\begin{array}{l}\text { Homogeneous random number } \\
\text { satisfying the condition } 0 \leq \\
p . d i r \leq \varphi\end{array}$ \\
\hline $\begin{array}{l}\text { Distance between } \\
\text { shooting location } \\
p . l o c \\
\text { viewpoint } g\end{array}$ & $\begin{array}{l}\text { Normal random number that } \\
\text { and } \\
\text { the distribution } \rho \sigma r_{s}\end{array}$ \\
\hline
\end{tabular}

Table 2. Generation condition of location - direction - enabled photograph $p$
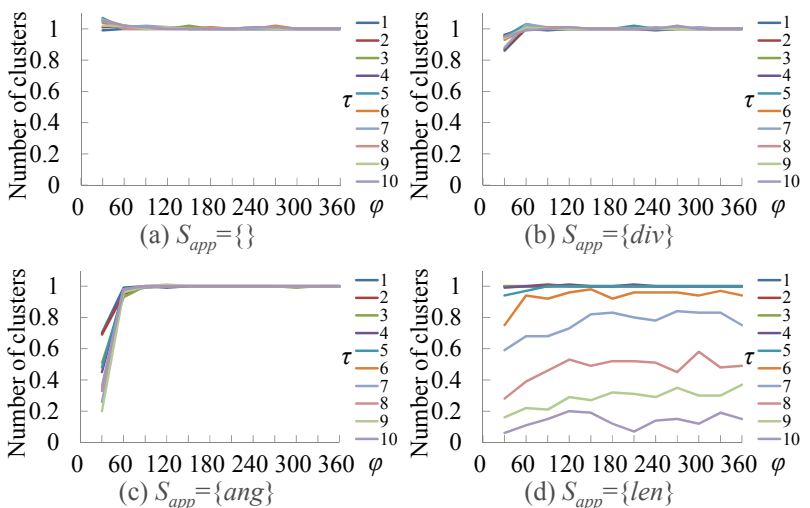

(b) $S_{a p p}=\{d i v\}$

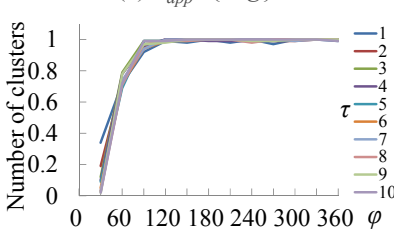
(e) $S_{\text {app }}=\{$ div, ang $\}$

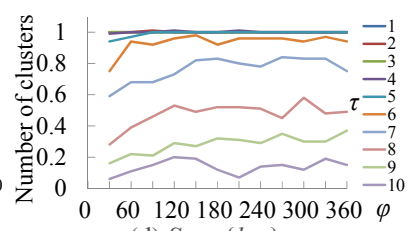
(d) $S_{a p p}=\{l e n\}$
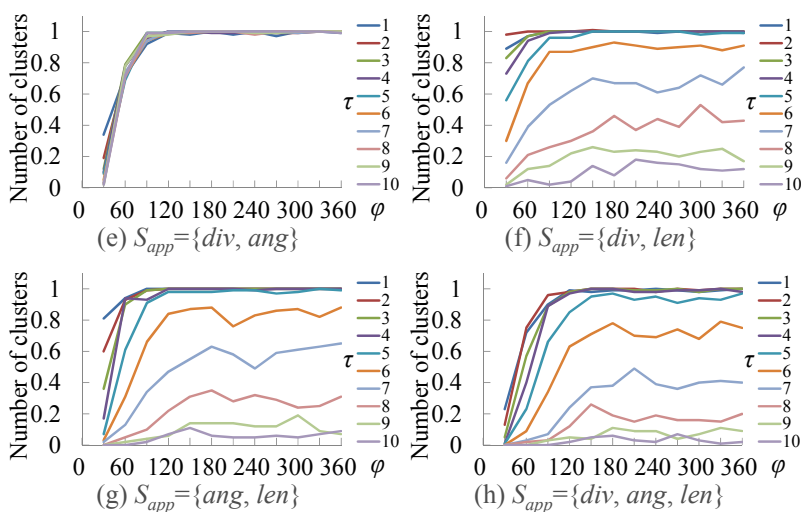

(f) $S_{\text {app }}=\{$ div, len $\}$

Figure 2. Average number of clusters per applied score

\section{Application example}

The details in spots of interest using dirDBSCAN (Eps, MinPts, $\sigma)=(4,4,10))$, and spots of viewpoint by DBSCAN $((E p s$, MinPts $)=(6,4))$ for the data collected from the large photo sharing service Flickr (1,008 photographs around Hibiya Park) are shown in Figure 3 and Table 3. The cluster ID is common to both the figure and the table. The terms without parentheses refer to spots of interest and those within parentheses refer to spots of viewpoint.

Different locations were extracted as clusters in dirDBSCAN and DBSCAN but there were also examples of overlapping locations, such as the arbor near the Kumogata Pond $(1,(\mathrm{~m}))$. Further, dirDBSCAN managed to extract certain clusters that could not have been done using existing methods. For instance, the locations of (a) a crane fountain and (b) a large fountain that cannot be entered as they are on water and in general, the shooting location and targeted place for the photograph did not coincide in such cases. It is a unique feat in the proposed method that these locations can be extracted.

In addition, connections were found between viewpoints and spots of interest that were close to each other. For example, there are spots dotted around the large fountain, which is a spot of interest, and viewpoints of especially high-density, for instance a place where the Hibiya Public Hall is captured in the background, which is where classic photos can be shot. 


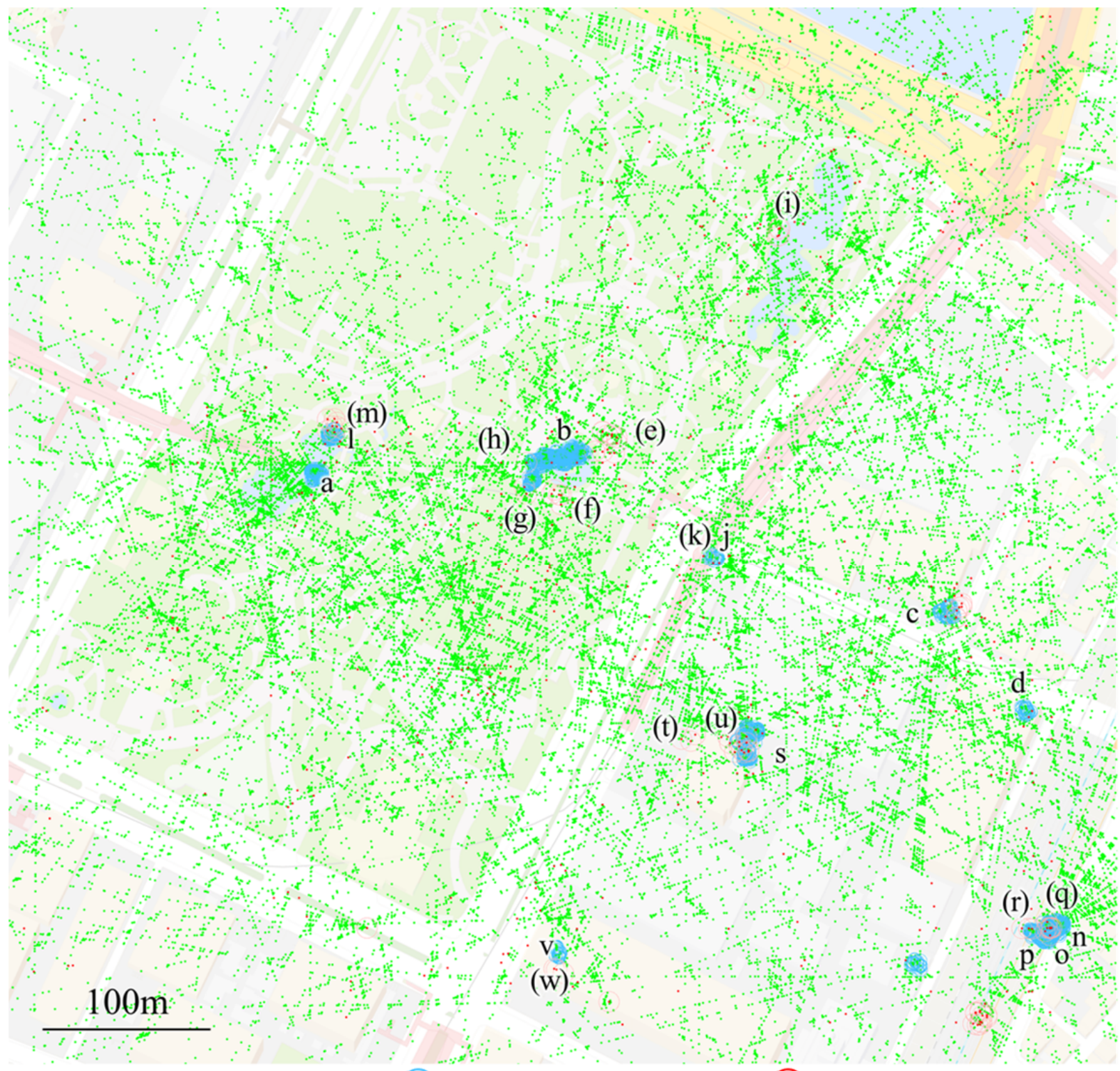
viewpoint
intersection
(a), (b)...spot of interest
$a, b, \ldots$ spot of viewpoint

Figure 3. Cluster extraction result by proposed method

\begin{tabular}{l|l}
\hline Spot target & cluster ID (terms in parentheses are spots of viewpoints) \\
\hline Spot of interest & $\mathrm{a}$ \\
\hline Crane fountain (Kumogata Pond) & $\mathrm{b}$ \\
\hline Large fountain (Fountain plaza) & $\mathrm{c}$ \\
\hline Tokyo Takarazuka Theater (Front) & $\mathrm{d}$ \\
\hline Imperial Hotel (Tower Side) & (e) (f) (g) (h) \\
\hline Spot of viewpoint & (i) \\
\hline Around the large fountain (4 places) & interest: $\mathrm{j}$, viewpoint: $(\mathrm{k})$ \\
\hline Bank of the Shinji Pond & interest: l, viewpoint: $(\mathrm{m})$ \\
\hline Places where Spot of interest and spot of viewpoint overlap \\
\hline Hibiya Gate intersection & interest: $\mathrm{n}$ o p, viewpoint: $(\mathrm{q})(\mathrm{r})$ \\
\hline Arbor (Kumagata Pond bank) & interest: $\mathrm{s}$, viewpoint: $(\mathrm{t})(\mathrm{u})$ \\
\hline Corridor Street & interest $\mathrm{v}$, viewpoint: $(\mathrm{w})$ \\
\hline Imperial Hotel (Park side) &
\end{tabular}

Table 3. Characteristic spots measured and extracted 


\section{Conclusions and future work}

We proposed a clustering method for directional data points and performed hot spot extraction from a set of location-direction-enabled photographs.

Future work includes considerations on accuracy of the location information.

\section{References}

Ester, M., Kriegel, H. P., Sander, J., Xu, X. (1996) A Density-Based Algorithm for Discovering Clusters in Large Spatial Databases with Noise. KDD 1996, 226231. 